\title{
Barley Stripe Mosaic Virus
}

\author{
Mahdi Tajalifar* \\ Plant Genetics and Breeding, Islamic Azad University, Takestan
}

${ }^{*}$ Corresponding author: Mahdi Tajalifar, PhD Student, Plant Genetics and Breeding, Islamic Azad University, Takestan; Tel: 0098-28-33367725; Email: Mahdi.tajalifar@yahoo.com

Received: February 08, 2022; Accepted: February 15, 2022; Published: February 18, 2022

\begin{abstract}
The aim of this study was to express TaSTP13 in wheat leaf by (pst). Some abnormal behaviors of TaSTP13 are concentrated in the plasma membrane and act as a homoligomer. Seizure of TaSTP13 reduces wheat susceptibility to Pst by gene silencing (VIGS) by barley bar mosaic virus. Molecular mechanisms and regulatory genes are needed to improve tolerance to environmental stress in products. The new 4-component version (with very high expression) and the 3-component version (with low expression) contain a barley mosaic virus (BSMV) virus-based system, which is used for the functional characterization of finger protein on the $\mathrm{C} 2 \mathrm{H} 2$ type in wheat. The four-component version, which contains a system based on the barley bar mosaic virus, has a high load capacity for the rapid and stable expression of recombinant proteins in various plant species. Excessive expression of TaSTP13 increases the susceptibility of Arabidopsis to powdery mildew and leads to increased glucose accumulation in the leaves.
\end{abstract}

Keywords: Homo ligomer, Gene silencing, Zinc finger protein and Arabidopsis

\section{Introduction}

The aim of this study was to express TaSTP13 in wheat leaf by Puccinia striiformis f.sp.tritici (pst). For example, is TaSTP13 involved in wheat susceptibility to rust and mildew? Is the decrease in glucose uptake related to wheat resistance due to Lr67? In this study, the expression of TaSTP13 homologues was specifically induced in wheat leaves challenged by the Pst pathotype, CYR31, and abiotic treatments. Intracellular localization analysis shows that TaSTP13 is located in the plasma membrane. Some of the abiotic behaviors of TaSTP13 are concentrated in the plasma membrane and act as hemoligomers [1-4].

To increase crop yield, genetic and molecular mechanisms that have mechanisms to withstand various environmental stresses should be increased in products. Most genomic research has focused on plant models or products with diploid genomes (such as Arabidopsis and Oryza sativa) [1].

To improve crop performance, special attention should be paid to the underlying genetic and molecular mechanisms of responses and mechanisms for tolerating various abiotic stresses in products [1].

Gene function is further manifested by transgenic gene expression or gene silencing in transgenic products. This process (gene function) in cereals is a time-consuming method. Plant viral expression systems can be used to rapidly express proteins [2].

BSMV virus is a triple-positive (alfa, beta, gama) stranded RNA virus with RNAs coated at the end of ' 5 (3). A barley mosaic virus (BSMV) virus-based gRNA delivery system targets mutant, wheat, and corn mutants for short, regular, clustered cross-repetitive palindromic replication (CRISPR)/cas9 [5].
The gRNA maintains the Cas9 interaction structure and the ability to identify target genes. In plants, gRNAs direct the Cas9 protein to cut double-stranded target DNA in cells to create double-stranded fractures that can be amplified by a non-identical end-binding pathway and/or a matched repair pathway for repair or mutation. Gives target genes [5].

\section{Material and Method}

The three- or four-component barley mosaic virus system is used to produce wheat. Functional properties of finger transcription factor on $\mathrm{C} 2 \mathrm{H} 2$ type in response to wheat environmental stress using the new 4-component BSMV system (with very high expression) and the three-component BSMV system is focused on gene regulation. To enhance the expression profile of TaSTP13, qRT-PCR measurement system is used.

\section{Conclusion}

The TaSTP13 gene is a protein encoder that carries sugar for wheat. Some of the abiotic behaviors of TaSTP13 are concentrated in the plasma membrane and act as hemoligomers.

\section{References}

1. Cheuk A, Ouellet F, Houde M (2020) The barley stripe mosaic virus expression system reveals the wheat $\mathrm{C} 2 \mathrm{H} 2$ zinc finger protein TaZFP1B as a key regulator of drought tolerance. BMC Plant Biology 20: 144.

2. Cheuk A, Houde M (2018) A New Barley Stripe Mosaic Virus Allows Large Protein Overexpression for Rapid Function Analysis. Plant Physiol 176: 1919-1931. [crossref]

3. Cheuk A, Houde M (2017) A rapid and efficient method for uniform gene expression using the barley stripe mosaic virus. Plant method 13: 24. [crossref] 
Mahdi Tajalifar (2022) Barley Stripe Mosaic Virus

4. Huai B, Yang Q, Wei X, Pan Q, Kang Z, et al. (2020) TaSTP13 contributes to wheat susceptibility to stripe rust possibly by increasing cytoplasmic hexose concentration. BMC Plant Biology 20: 49. [crossref]
5. Hu J, Li s, Li H, Li Z, Song W, et al. (2019) A barley stripe mosaic virus-based guide RNA delivery system for targeted mutagenesis in wheat and maize. Mol Plant Pathol 20: 1463-1474. [crossref]

\section{Citation:}

Tajalifar M (2022) Barley Stripe Mosaic Virus. Mol Genet Res Open Volume 5(1): 1-2. 